\title{
VALUE DISTRIBUTION OF HARMONIC POLYNOMIALS IN SEVERAL REAL VARIABLES $\left(^{1}\right)$
}

\author{
BY \\ MORRIS MARDEN
}

\begin{abstract}
Using Bergman's integral operator method, the author studies an arbitrary axisymmetric harmonic polynomial $H(x, \rho)$ in $R^{3}$ and $R^{N}$ in relation to its associate polynomial $h(\zeta)$ in $C$. His results pertain to the value distributions and critical circles of $H(x, \rho)$ in certain cones; bounds on the gradient of an $H(x, \rho)$ assumed bounded in sphere $x^{2}+\rho^{2} \leqq 1$; axisymmetric harmonic vectors. Corresponding results are also obtained for axisymmetric harmonic functions $F(x, \rho)$ with rational associate $f(\zeta)$.
\end{abstract}

1. Introduction. In this paper we study axisymmetric harmonic polynomials and certain other harmonic functions in several real variables. Among the properties we investigate are the following: the location of points where assigned values are assumed; coincidence theorems; the location of the critical points; and the behavior of the gradient of bounded harmonic polynomials.

Since we deal largely with entities having axial symmetry, we need not only rectangular coordinates $(x, y, z)$, but also cylindrical coordinates $(x, \rho, \phi)$ with

$$
\rho^{2}=y^{2}+z^{2}, \quad y=\rho \cos \phi, \quad z=\rho \sin \phi ;
$$

and spherical coordinates $(r, \theta, \phi)$ with

$$
\rho=r \sin \theta, \quad x=r \cos \theta .
$$

By an axisymmetric function we mean one that is independent of $\phi$.

It is well known that every axisymmetric harmonic polynomial $H(x, \rho)$ of degree $n$ can be represented $[6$, p. 254] in the form

$$
H(x, \rho)=\sum_{k=0}^{n} a_{k} r^{k} P_{k}(x / r)
$$

Presented to the Society July 20, 1970; received by the editors July 21, 1970.

AMS 1970 subject classifications. Primary 31B05, 44A15; Secondary 30A08, 30A70.

Key words and phrases. Axisymmetric harmonic polynomials, Bergman integral method, analytic theory of polynomials, coincidence theorems, axisymmetric harmonic functions, gradient of bounded harmonic polynomials, Bernstein's Theorem, critical circles of axisymmetric harmonic polynomials, axisymmetric harmonic vectors, axisymmetric flow potential, Stoke's stream function.

(1) This paper was written partly under NSF grant GP-19615 and announced in Abstract 70T-B203, Notices Amer. Math. Soc. 17 (1970), 592-593.

Copyright (C) 1971, American Mathematical Society 
where $P_{k}(u)$ is the Legendre polynomial of degree $k$. Let us define as the associate of $H(x, \rho)$ the polynomial

$$
h(\zeta)=\sum_{k=0}^{n} a_{k} \zeta^{k}
$$

of the single complex variable $\zeta=\xi+i \eta$. In view of the Whittaker formula [5, pp. 312-315]

$$
r^{k} P_{k}(x / r)=(1 / 2 \pi) \int_{0}^{2 \pi}(x+i \rho \cos t)^{k} d t
$$

we may represent $H(x, \rho)$ in terms of $h(\zeta)$ as

$$
H(x, \rho)=(1 / 2 \pi) \int_{0}^{2 \pi} h(x+i \rho \cos t) d t .
$$

More generally, let $f(\zeta)$ be holomorphic in an axiconvex region $\omega \subset C$, meaning a region such that $\zeta \in \omega$ implies $\lambda \zeta+(1-\lambda) \xi \in \omega$ for all $\lambda$ with $0 \leqq \lambda \leqq 1$. Then, in the axisymmetric region $\Omega$ obtained on rotating $\omega$ about the axis of reals,

$$
F(x, \rho)=(1 / 2 \pi) \int_{0}^{2 \pi} f(x+i \rho \cos t) d t
$$

is an axisymmetric harmonic function. In fact, (1.4) is the special case of the operator introduced by Bergman [1, p. 43]

$$
F(x, y, z)=(1 / 2 \pi i) \int_{|\tau|=1} f(\zeta, \tau) \tau^{-1} d t
$$

where $f(\zeta, \tau)$ is a function which is holomorphic in $\zeta$ over some region in $C$ and is continuous in $\tau$ for $|\tau|=1$. With

$$
\zeta=x+\frac{1}{2}(y i+z) \tau+\frac{1}{2}(y i-z) \tau^{-1},
$$

the operator (1.5) transforms $f(\zeta, \tau)$ into the function $F(x, y, z)$ which together with its real and imaginary parts is harmonic in a certain region of $R^{3}$.

Bergman has successfully applied this method to the study of various properties of harmonic functions $F(x, y, z)$, particularly the singularities of $F(x, y, z)$ in relation to the singularities of the associate function $f(\zeta, \tau)$. Furthermore, to learn the effect of the zeros of $f(\zeta, \tau)$ upon $F(x, y, z)$, he has suggested the determination of the singularities of the harmonic function $\Phi(x, y, z)$ having $\phi(\zeta, \tau)=1 / f(\zeta, \tau)$ as its associate. However, results so obtained regarding $\Phi$ are difficult to interpret in terms of $F$ since no simple relation exists between $\Phi$ and $F$.

While the latter indirect procedure may be unavoidable in the general case, direct approaches are possible and will be introduced here in the special cases of axisymmetric and certain other harmonic polynomials in $R^{N}$. In these special cases, we propose to use some simple methods drawn from the analytic theory of 
polynomials (in one complex variable) in order to study directly the influence of the zeros (or value distribution) of $f(\zeta, \tau)$ upon the behavior of $F(x, y, z)$.

2. Value distributions of axisymmetric harmonic polynomials. A result that follows immediately from (1.1) or from (1.3) is that $H(x, 0)=h(x)$. That is, an axisymmetric harmonic function assumes the same values as its associate on the axis of symmetry.

More generally, we shall now prove that an axisymmetric harmonic polynomial omits a given value in a certain cone if its associate polynomial omits the same value in a corresponding sector.

THEOREM 2.1. Let $H(x, \rho)$ be an axisymmetric harmonic polynomial of degree $n$ and let $h(\zeta)$ be its associate. Let $\gamma$ and $c$ be arbitrary real constants. If $h(\zeta) \neq \gamma$ in the sector

$$
|\arg (\zeta-c)|<\pi-(\pi / 2 n),
$$

then $H(x, \rho) \neq \gamma$ in the cone

$$
0<\rho \leqq(x-c) \tan (\pi / 2 n) .
$$

REMARK. We may take $\gamma$ complex in this and subsequent theorems when Im $H(x, \rho) \not \equiv 0$.

Proof. Let us denote by $\alpha_{1}, \alpha_{2}, \ldots, \alpha_{n}$ the points in $C$ where $h(\zeta)=\gamma$. Then

$$
h(\zeta)-\gamma=a_{n} \prod_{j=1}^{n}\left(\zeta-\alpha_{j}\right),
$$

$$
H(x, \rho)-\gamma=(1 / 2 \pi) \int_{0}^{2 \pi}[h(x+i \rho \cos t)-\gamma] d t .
$$

Let us suppose that $H(x, \rho)$ assumes the value $\gamma$ on the circle $x=x_{0}, \rho=\rho_{0}$ in the cone (2.2). Then

$$
\int_{0}^{2 \pi} w(t) d t=0, \quad w(t)=\prod_{j=1}^{n}\left(\alpha_{j}-x_{0}-i \rho_{0} \cos t\right) .
$$

Since the point $\zeta=x_{0}+i \rho_{0}$ lies in the sector $-\pi / n<\arg (\zeta-c)<\pi / n$, so does the point $\zeta=x_{0}+i \rho_{0} \cos t$ for all values of $t$.

Since, by hypothesis, $\pi-(\pi / 2 n) \leqq \arg \left(\alpha_{j}-c\right) \leqq \pi+(\pi / 2 n)$, the ray from each $\alpha_{i}$ to $x_{0}+i \rho_{0} \cos t$ lies interior to the sector

$$
\pi-(\pi / 2 n)<\arg \left(\zeta-x_{0}-i \rho_{0} \cos t\right)<\pi+(\pi / 2 n) .
$$

Hence,

$$
\pi-(\pi / 2 n)<\arg \left(\alpha_{j}-x_{0}-i \rho_{0} \cos t\right)<\pi+(\pi / 2 n),
$$

for each $j, j=1,2, \ldots, n$, and each $t, 0 \leqq t \leqq 2 \pi$. Therefore, from (2.5) and (2.6) follows that

$$
n \pi-(\pi / 2)<\arg w(t)<n \pi+\pi / 2
$$


Thus the integral in (2.5), as the limit of a sum of vectors $w(t)$ drawn from the origin to points in the convex sector (2.7), cannot vanish contrary to (2.5). Hence, $H\left(x_{0}, \rho_{0}\right) \neq \gamma$ as was to be proved.

We shall now apply Theorem 2.1 to the case that the zeros of $h(\zeta)-\gamma$ are known to lie in a bounded region $\omega$ which is axiconvex. Let us denote by $c_{1}$ and $c_{2}$ the two points on the real axis, situated left and right of $\omega$, from which $\omega$ subtends an angle of $\pi / n$. Let us denote by $K_{1}(\omega, n)$ and $K_{2}(\omega, n)$ respectively the cones

$$
\begin{aligned}
& 0<\rho \leqq\left(c_{1}-x\right) \tan (\pi / 2 n), \\
& 0<\rho \leqq\left(x-c_{2}\right) \tan (\pi / 2 n) .
\end{aligned}
$$

We now shall deduce the following result.

THEOREM 2.2. Let $H(x, \rho)$ be an axisymmetric harmonic polynomial and $h(\zeta)$ its associate. Let $\omega \subset C$ be a bounded axiconvex region. If $h(\zeta) \neq \gamma$ when $\zeta \notin \omega$, then $H(x, \rho) \neq \gamma$ on any circle $x=x_{0}, \rho=\rho_{0}$ in cone $K_{1}(\omega, n)$ or cone $K_{2}(\omega, n)$.

REMARK. Let $\Omega$ denote the region obtained on revolving $\omega$ about the $x$-axis. If a double-nappe, axisymmetric cone with vertex angle of $\pi / n$ were moved as far as possible to the left with its left nappe always containing $\Omega$, the interior of the right nappe is the region $K_{2}(\omega, n)$.

Proof. The region $\omega$ lies in the intersection of the sectors

$$
\begin{gathered}
-\pi / 2 n<\arg \left(\zeta-c_{1}\right)<\pi / 2 n, \\
\pi-(\pi / 2 n)<\arg \left(\zeta-c_{2}\right)<\pi+(\pi / 2 n) .
\end{gathered}
$$

To establish Theorem 2.2, we need merely to apply Theorem 2.1 to each of two sections (2.10) and (2.11).

In the special case that $\omega$ is a disk, we can restate Theorem 2.2 as follows:

THEOREM 2.3. Let $H(x, \rho)$ be an axisymmetric harmonic polynomial of degree $n$ and $h(\zeta)$ its associate. Let $\gamma$ be an arbitrary real constant. If $h(\zeta) \neq \gamma$ for $|\zeta|>a$, then $H(x \rho,) \neq \gamma$ in the two cones

$$
0<\rho \leqq \pm x \tan (\pi / 2 n)-a \sec (\pi / 2 n) .
$$

We now restate Theorem 2.3 in terms of the coefficients of $H(x, \rho)$.

THEOREM 2.4. Let $H(x, \rho)$ be the axisymmetric harmonic polynomial

$$
H(x, \rho)=\sum_{j=0}^{n} a_{j} r^{j} P_{j}(x / r)
$$

and let $\gamma$ be an arbitrary real constant.

Then $H(x, \rho) \neq \gamma$ in the two cones (2.12) where

$$
a=1+\max \left\{\left|a_{0}-\gamma\right| /\left|a_{n}\right|,\left|a_{1} / a_{n}\right|, \ldots,\left|a_{n-1} / a_{n}\right|\right\} .
$$


Proof. Let us denote by $h(\zeta)$ the associate of $H(x, \rho)$. Then by Cauchy's wellknown inequality [3, p. 123], the zeros of

$$
h(\zeta)-\gamma=\left(a_{0}-\gamma\right)+a_{1} \zeta+a_{2} \zeta^{2}+\cdots+a_{n} \zeta^{n}
$$

satisfy the inequality $|\zeta| \leqq a$, with $a$ given by (2.14). If we now apply Theorem 2.3, we get at once Theorem 2.4 .

Alternatively we can restate Theorem 2.3 using the many other well-known bounds on the zeros of a polynomial in a complex variable [3, Chapter VII].

3. Coincidence theorems for axisymmetric harmonic polynomials. We now prove the following

THEOREM 3.1. Let $H(x, \rho)$ be an axisymmetric harmonic polynomial of degree $n$ and $h(\zeta)$ be its associate. Let $\Gamma$ be a circular domain. If $h(\zeta) \neq \gamma$ for $\zeta \notin \Gamma$, and if $x=x_{0}, \rho=\rho_{0}$ is a circle on which $H(x, \rho)=\gamma$, then there exists $\alpha \in \Gamma$ such that $x_{0}, \rho_{0}$ satisfy the equation

$$
\sum_{j=0}^{n}(-1)^{j}\left(\begin{array}{l}
n \\
j
\end{array}\right) \alpha^{n-j} r_{0}^{j} P_{j}\left(x_{0} / r_{0}\right)=0
$$

where $r_{0}^{2}=x_{0}^{2}+\rho_{0}^{2}$.

Proof. In Theorem 3.1, a circular domain $\Gamma$ means the interior or exterior of a circle or a half plane in $\boldsymbol{C}$.

Using equations (2.3) and (2.4), we infer that

$$
H\left(x_{0}, \rho_{0}\right)-\gamma=(1 / 2 \pi) \int_{0}^{2 \pi}\left[h\left(x_{0}+i \rho_{0} \cos t\right)-\gamma\right] d t=0 .
$$

This is a linear relation among the coefficients of polynomial $h(\zeta)$. Since all the zeros of $h(\zeta)-\gamma$ are in $\Gamma$, we may apply the well-known Grace's Theorem [3, pp. 62-63] according to which there exists a point $\alpha \in \Gamma$ such that

$$
H\left(x_{0}, \rho_{0}\right)-\gamma=(1 / 2 \pi) \int_{0}^{2 \pi} a_{n}\left(x_{0}+i \rho_{0} \cos t-\alpha\right)^{n} d t=0 .
$$

If we now expand the integrand and apply (1.2), we obtain equation (3.1) for $x_{0}$ and $\rho_{0}$.

REMARK. In view of (2.3), we may write as an equation for $x_{0}, \rho_{0}$ :

$$
a_{n}^{-1}\left[H\left(x_{0}, \rho_{0}\right)-\gamma\right]=\sum_{j=0}^{n}(-1)^{j} S_{n-j} r_{0}^{j} P_{j}\left(x_{0} / r_{0}\right)=0
$$

where $S_{k}$ is the sum of the products of the $\alpha_{j}$ taken $k$ at a time. If we cause the $\alpha_{j}$ as points in $\Gamma$ to coalesce at a suitable point $\alpha$ in $\Gamma$, we then obtain equation (3.1) from equation (3.4). In this sense, Theorem 3.1 is a "coincidence theorem".

From Theorem 3.1 we may now deduce at once the following result: 
THEOREM 3.2. Let $H(x, \rho)$ be an axisymmetric harmonic polynomial and $\gamma$ an arbitrary real constant. If $x=x_{0}, \rho=\rho_{0}$ is a circle along which $H(x, \rho)=\gamma$, then $x_{0}, \rho_{0}$ satisfy equation (3.1) where $\alpha$ is some point with $|\alpha| \leqq a$ and $a$ is given by (2.14).

From (3.1), we can also derive an inequality between $|\alpha|$ and $r_{0}$. For, writing (3.1) as

$$
\alpha^{n}=\sum_{j=1}^{n}(-1)^{j+1}\left(\begin{array}{l}
n \\
j
\end{array}\right) \alpha^{n-j} r_{0}^{j} P_{j}\left(x_{0} / r_{0}\right)
$$

and using the fact that $\left|P_{j}(\cos \theta)\right| \leqq 1$, we infer that

$$
|\alpha|^{n} \leqq \sum_{j=1}^{n}\left(\begin{array}{c}
n \\
j
\end{array}\right)|\alpha|^{n-j} r_{0}^{j}=\left(|\alpha|+r_{0}\right)^{n}-|\alpha|^{n}
$$

Hence,

$$
\left(2^{1 / n}-1\right)|\alpha| \leqq r_{0}=\left\{x_{0}^{2}+\rho_{0}^{2}\right\}^{1 / 2}
$$

Likewise

$$
r_{0}^{n}\left|P_{n}\left(x_{0} / r_{0}\right)\right| \leqq \sum_{j=0}^{n-1}\left(\begin{array}{l}
n \\
j
\end{array}\right)|\alpha|^{n-j} r_{0}^{j}=\left(|\alpha|+r_{0}\right)^{n}-r_{0}^{n}
$$

so that, choosing $x_{0}=r_{0}$ and noting $P_{n}(1)=1, r_{0}\left[2^{1 / n}-1\right] \leqq|\alpha|$. In short,

$$
\left(2^{1 / n}-1\right) r_{0} \leqq|\alpha| \leqq r_{0}\left[2^{1 / n}-1\right]^{-1} \text {. }
$$

In the above treatment we have expressed the right side of (3.3) in terms of the Legendre polynomials of a real variable. On admitting Legendre polynomials of a complex variable, we are able to interpret (3.3) immediately as follows due to (1.2).

THEOREM 3.3. Let $H(x, \rho)$ be an axisymmetric harmonic polynomial whose associate $h(\zeta)$ assumes a given real value $\gamma$ only in a circular domain $\Gamma \subset C$. Then corresponding to any circle $x=x_{0}, \rho=\rho_{0}$ for which $H\left(x_{0}, \rho_{0}\right)=\gamma$, there exists $\alpha \in \Gamma$ such that $\left(x_{0}, \rho_{0}\right)$ satisfies the equation

$$
P_{n}\left(\lambda\left(x_{0}-\alpha\right)\right)=0
$$

where $\lambda$ is a suitable branch of the function $\lambda=\left[\left(x_{0}-\alpha\right)^{2}+\rho_{0}^{2}\right]^{-1 / 2}$.

Theorem 3.3 is more obviously a coincidence theorem in the sense that equation (3.5) involving only one Legendre polynomial may be regarded as a condensation of the equation $H\left(x_{0}, \rho_{0}\right)-\gamma=0$ involving $n+1$ Legendre polynomials.

4. Value distribution of axisymmetric harmonic functions with rational associate. We shall now use the methods of $\S 2$ to explore the properties of harmonic functions

$$
\Psi(x, \rho)=(1 / 2 \pi) \int_{0}^{2 \pi} \psi(x+i \rho \cos t) d t
$$

where $\psi(\zeta)$ is a rational function. Whereas a polynomial $\psi(\zeta)$ implied a polynomial $\Psi(x, \rho)$, a rational $\psi(\zeta)$ does not in general lead to a rational harmonic function 
$\Psi(x, \rho)$ but poles of $\psi(\zeta)$ may correspond to certain singularities of $\Psi(x, \rho)$ [1, pp. 43-49].

We begin with the following theorem:

THEOREM 4.1. Let $\Psi(x, \rho)$ be an axisymmetric harmonic function whose associate $\psi(\zeta)$ is a rational function which has $n$ finite poles (counted with multiplicity). Let $\gamma$ be an arbitrary real finite constant such that $\psi(\zeta) \neq \gamma$ in the sector

$$
|\arg (\zeta-c)| \leqq \pi-(\pi / 2 \mu)
$$

where $\mu=m+n$ and where $m$ is the number (counted with multiplicity) of finite points $\alpha_{j}$ for which $\psi\left(\alpha_{j}\right)=\gamma$. If also no finite poles of $\psi$ lie in sector (4.2), then $\Psi(x, \rho) \neq \gamma$ in the cone

$$
0<\rho \leqq(x-c) \tan (\pi / 2 \mu) .
$$

Proof. Let us write $\psi(\zeta)=p(\zeta) / q(\zeta)$ where $p(\zeta)$ and $q(\zeta)$ are polynomials, and further write

$$
\begin{gathered}
p(\zeta)-\gamma q(\zeta)=a_{n} \prod_{j=1}^{m}\left(\zeta-\alpha_{j}\right), \\
q(\zeta)=b_{n} \prod_{j=1}^{n}\left(\zeta-\beta_{j}\right) .
\end{gathered}
$$

From (4.1), (4.4) and (4.5), we get for any circle $x=x_{0}, \rho=\rho_{0}$ for which $\Psi\left(x_{0}, \rho_{0}\right)$ $=\gamma$, the expression

$$
\begin{gathered}
\int_{0}^{2 \pi} w(t) d t=0 \\
\bar{w}(t)=\prod_{j=1}^{m}\left(\alpha_{j}-x_{0}-i \rho_{0} \cos t\right) / \prod_{j=1}^{n}\left(\beta_{j}-x_{0}-i \rho_{0} \cos t\right) .
\end{gathered}
$$

By hypotheses all the $\alpha_{j}$ and $\beta_{j}$ lie in the complement of sector (4.2) and so satisfy the inequalities

$$
\pi-(\pi / 2 \mu)<\arg \left(\zeta-x_{0}-i \rho_{0} \cos t\right)<\pi+(\pi / 2 \mu)
$$

for all $j$ and for all $t$. Hence, from (4.7),

$$
(m-n) \pi-(\pi / 2)<\arg w(t)<(m-n) \pi+\pi / 2 .
$$

As in the proof of Theorem 2.1, we now conclude that the integral in (4.6) cannot vanish. That is, for every circle $x=x_{0}, \rho=\rho_{0}$ lying in the cone (4.3), we have $\Psi\left(x_{0}, \rho_{0}\right) \neq \gamma$ as was to be proved.

In analogy with Theorem 2.2, we may state the following

THEOREM 4.2. Let $\Psi(x, \rho)$ be an axisymmetric harmonic function whose associate $\psi(\zeta)$ is a rational function. Let $\omega$ be a bounded axiconvex region that contains the finite poles of $\psi$ as well as the finite points where $\psi(\zeta)=\gamma$. Then $\Psi(x, \rho) \neq \gamma$ along any 
circle $x=x_{0}, \rho=\rho_{0}$ contained in either cone $K_{j}(\omega, m+n), j=1$ or 2 , where $m$ and $n$ are the numbers of finite points, counted with multiplicity, at which $\psi(\zeta)=\gamma$ and $\psi(\zeta)=\infty$ respectively.

Instead of assuming that the finite zeros and poles of the associate are in the same region $\omega$, we shall now suppose that they lie in separate disjoint regions.

THEOREM 4.3. Let $\omega_{1}$ and $\omega_{2}$ be two bounded axiconvex regions, such that $\mathscr{R}\left(\zeta_{1}\right)<\mathscr{R}\left(\zeta_{2}\right)$ for all $\zeta_{1} \in \omega_{1}$ and $\zeta_{2} \in \omega_{2}$. Let $\Psi(x, \rho)$ be an axisymmetric harmonic function with rational associate $\psi(\zeta)$. Assume that counted with multiplicity all $m$ finite points where $\psi(\zeta)=\gamma$ lie in $\omega_{1}$ and all $n$ finite poles of $\psi$ lie in $\omega_{2}$. Assume further that in the notation of Theorem 2.2 .

$$
\Omega=K_{2}\left(\omega_{1}, \mu\right) \cap K_{1}\left(\omega_{2}, \nu\right) \neq \varnothing
$$

for some choice of positive numbers $\mu$ and $\nu$ such that

$$
(m / \mu)+(n / v)<1 .
$$

Then $\Psi(x, \rho) \neq \gamma$ along any circle $x=x_{0}, \rho=\rho_{0}$ which lies in the region $\Omega$.

Proof. If $x=x_{0}, \rho=\rho_{0}$ is a circle in $\Omega$ along which $\Psi(x, \rho)=\gamma$, the points $\alpha_{j}$ defined in (4.4) satisfy the inequality (4.8), whereas the points $\beta_{j}$ defined in (4.5) satisfy the inequality

$$
-\pi / 2 \nu<\arg \left(\zeta-x_{0}-i \rho_{0} \cos t\right)<\pi / 2 \nu .
$$

Hence, $w(t)$ defined in (4.7) satisfies the inequality $m \pi-(m \pi / 2 \mu)-(n \pi / 2 \nu)$ $<\arg w(t)<m \pi+(m \pi / 2 \mu)+(n \pi / 2 \nu)$. Due to (4.10) vector $w(t)$ remains in the same convex sector as $t$ varies and so integral (4.6) cannot vanish. This means that $\Psi(x, \rho) \neq \gamma$ along all circles $x=x_{0}, \rho=\rho_{0}$ in $\Omega$, thus establishing Theorem 4.3.

5. Derivatives of axisymmetric harmonic polynomials. In this section we try to construct some analogues to the following well-known theorem of S. Bernstein $[4$, p. 45].

Let the nth degree polynomial $h(\zeta)$ have the property that $|h(\zeta)| \leqq 1$ for $|\zeta| \leqq 1$. Then its derivative has the property that $\left|h^{\prime}(\zeta)\right| \leqq n$ for $|\zeta| \leqq 1$, this bound being attained by polynomials $h(\zeta)=\lambda \zeta^{n}$ if $|\lambda|=1$.

We shall now show that the radial derivative of $H(x, \rho)$ satisfies a similar inequality, as does the axial derivative.

THEOREM 5.1. Let $H(x, \rho)$ be an axisymmetric harmonic polynomial of degree $n$ whose associate $h(\zeta)$ satisfies the relation

$$
|h(\zeta)| \leqq 1 \text { for }|\zeta| \leqq 1
$$

Then $|H(x, \rho)| \leqq 1$ and

$$
|\partial H / \partial x| \leqq n, \quad|\partial H / \partial r| \leqq n \text { for } r^{2}=x^{2}+\rho^{2} \leqq 1,
$$


the bounds being attained by

$$
H(x, \rho)=r^{n} P_{n}(x / r) .
$$

Proof. Writing $H(x, \rho)$ in the form (1.3), we find

$$
\begin{aligned}
& \partial H / \partial x=(1 / 2 \pi) \int_{0}^{2 \pi} h^{\prime}(x+i \rho \cos t) d t, \\
& \partial H / \partial \rho=(i / 2 \pi) \int_{0}^{2 \pi} h^{\prime}(x+i \rho \cos t) \cos t d t .
\end{aligned}
$$

Thus

$$
\begin{aligned}
\partial H / \partial r & =(x / r)(\partial H / \partial x)+(\rho / r)(\partial H / \partial \rho) \\
& =(1 / 2 \pi r) \int_{0}^{2 \pi} h^{\prime}(x+i \rho \cos t)(x+i \rho \cos t) d t .
\end{aligned}
$$

Applying Bernstein's Theorem, we find

$$
\begin{aligned}
|\partial H / \partial r| & \leqq(n / 2 \pi) \int_{0}^{2 \pi}\left[(x / r)^{2}+(\rho / r)^{2} \cos ^{2} t\right]^{1 / 2} d t \\
& \leqq(2 n / \pi) \int_{0}^{\pi / 2}\left[1-(\rho / r)^{2} \sin ^{2} t\right]^{1 / 2} d t \\
& \leqq(2 n / \pi) E(\rho / r) \leqq n
\end{aligned}
$$

where $E(k)$ is the complete elliptic integral of modulus $k$.

In the special case (5.3), we obtain from (5.6)

$$
\partial H / \partial r=(n / 2 \pi r) \int_{0}^{2 \pi}(x+i \rho \cos t)^{n} d t=(n / r) r^{n} P_{n}(x / r) .
$$

Hence, for $x=r=1$, we find $\partial H / \partial r=n$ so that the bound in (5.2) is attained by (5.3). The same is true for $\partial H / \partial x$.

We now turn to the gradient vector

$$
\nabla H=(\partial H / \partial x) i_{x}+(\partial H / \partial \rho) i_{\rho}
$$

where $i_{x}$ and $i_{\rho}$ are the unit vectors in the positive $x$ and $\rho$ directions respectively. For $|\nabla H|$ we propose the following theorem:

THEOREM 5.2. Let $H(x, \rho)$ be an axisymmetric harmonic polynomial of degree $n$ whose associate $h(\zeta)$ satisfies condition (5.1). Then its gradient vector $\nabla H$ has the property

$$
|\nabla H| \leqq n\left[1+(2 / \pi)^{2}\right]^{1 / 2}<1.1855 n
$$

for $r^{2}=x^{2}+\rho^{2} \leqq 1$. If, however,

$$
H(x, \rho)=\sum_{k=0}^{n} a_{k} r^{k} P_{k}(x / r), \quad \sum_{k=0}^{n}\left|a_{k}\right| \leqq 1,
$$

then $|\nabla H| \leqq n$, the bound being attained by $\lambda r^{n} P_{n}(x / r),|\lambda|=1$. 
Proof. Applying Bernstein's Theorem to (5.4) and (5.5), we learn that

$$
|\partial H / \partial x| \leqq n ; \quad|\partial H / \partial \rho| \leqq(n / 2 \pi) \int_{0}^{2 \pi}|\cos t| d t=2 n / \pi .
$$

Since $|\nabla H|^{2}=(\partial H / \partial x)^{2}+(\partial H / \partial \rho)^{2}$, we thus obtain (5.7).

In case of polynomial (5.3), we find

$$
\begin{aligned}
\partial H / \partial x & =r^{n-1}\left[n \cos \theta P_{n}(\cos \theta)+\sin ^{2} \theta P_{n}^{\prime}(\cos \theta)\right], \\
\partial H / \partial \rho & =r^{n-1}(\sin \theta)\left[n P_{n}(\cos \theta)-\cos \theta P_{n}^{\prime}(\cos \theta)\right], \\
|\nabla H|^{2} & =r^{2(n-1)}\left\{n^{2}\left[P_{n}(\cos \theta)\right]^{2}+\left[(d / d \theta) P_{n}(\cos \theta)\right]^{2}\right\} .
\end{aligned}
$$

Substituting into (5.9) the well-known form [see 6, p. 128]

$$
P_{n}(\cos \theta)=\sum_{j=0}^{[n / 2]} c_{j} \cos (n-2 j) \theta, \quad c_{j}>0,
$$

we find that

$$
\begin{aligned}
-(d / d \theta) P_{n}(\cos \theta) & =\sum_{j=0}^{[n / 2]}(n-2 j) c_{j} \sin (n-2 j) \theta, \\
\left(r^{1-n}|\nabla H|\right)^{2} & =\sum_{j=0}^{[n / 2]} c_{j}^{2} A_{j}(\theta)+2 \sum_{j=0}^{[n / 2]} \sum_{k=j+1}^{[n / 2]} c_{j} c_{k} B_{j k}(\theta)
\end{aligned}
$$

where $[n / 2]$ denotes the largest integer not exceeding $n / 2$,

$$
\begin{aligned}
A_{j}(\theta)= & (n-2 j)^{2}+4 j(n-j) \cos ^{2}(n-2 j) \theta, \\
B_{j k}(\theta)= & (n-2 j)(n-2 k) \cos 2(j-k) \theta \\
& +[2 j(n-k)+2 k(n-j)] \cos (n-2 j) \theta \cos (n-2 k) \theta .
\end{aligned}
$$

Since $A_{j}(\theta) \leqq A_{j}(0), B_{j k}(\theta) \leqq B_{j k}(0),(\partial H / \partial x)_{\theta=0}=r^{n-1} n P_{n}(1)$ and $[\partial H / \partial \rho]_{\theta=0}=0$, it follows that

$$
\left(r^{1-n}|\nabla H|\right)^{2} \leqq\left(r^{1-n}|\nabla H|\right)_{\theta=0}^{2}=n^{2} P_{n}(1)=n^{2} .
$$

Applying this result to (5.8), we learn that

$$
|\nabla H| \leqq \sum_{k=0}^{n} k\left|a_{k}\right| \leqq n
$$

the equality sign holding only if $a_{0}=a_{1}=\cdots=a_{n-1}=0,\left|a_{n}\right|=1$. This completes the proof of Theorem 5.2.

6. Critical circles of axisymmetric harmonic polynomials. The critical circles of $H(x, \rho)$ are those with equations $x=x_{0}, \rho=\rho_{0}$ that satisfy the relation

$$
\nabla H=0 \text { for } x=x_{0}, \rho=\rho_{0} .
$$


It is most convenient here to require that $x=x_{0}, \rho=\rho_{0}$ satisfy simultaneously the equations equivalent to $(6.1)$ :

$$
\partial H / \partial x=0, \quad \partial H / \partial r=0,
$$

where $\partial H / \partial x$ and $\partial H / \partial r$ are given by (5.4) and (5.6).

An immediate result is the following concerning critical circles that degenerate into single points.

THEOREM 6.1. Let $H(x, \rho)$ be an axisymmetric harmonic polynomial and let $h(\zeta)$ be its associate. If the real number $\zeta=x_{0}$ is a critical point of $h(\zeta)$, then also $x=x_{0}$, $\rho=0$ is a critical point of $H(x, \rho)$.

In the more general case, let us establish the following result:

THEOREM 6.2. Let $H(x, \rho)$ be an axisymmetric harmonic polynomial of degree $n$ and let $h(\zeta)$ be its associate. In $C$ let $\kappa^{\prime}$ denote a convex region symmetric in the real axis and containing all the critical points $\left(b_{1}, b_{2}, \ldots, b_{n-1}\right)$ of $h(\zeta)$ and let $\kappa_{0}^{\prime}$ be the convex hull of set $\left\{0, \kappa^{\prime}\right\}$. Then in the notation of Theorem 2.2, no locus $x=x_{0}, \rho=\rho_{0}$ is a critical circle of $H(x, \rho)$ if this circle lies in any of the cones $K_{j}\left(\kappa^{\prime}, n-1\right)$, $K_{j}\left(\kappa_{0}^{\prime}, n\right), j=1$ or 2 .

Proof. Equations (5.4) and (5.6) say in effect that $\partial H / \partial x$ and $r \partial H / \partial r$ are axisymmetric harmonic polynomials which are respectively of degrees $n-1$ and $n$ and of which the associates are respectively $h^{\prime}(\zeta)$ and $\left[\zeta h^{\prime}(\zeta)\right]$. To obtain the proof of Theorem 6.2, we apply Theorem 2.2 to each of the functions $\partial H / \partial x$ and $r \partial H / \partial r$ with $\gamma=0$ and use the fact that a critical circle $x=x_{0}, \rho=\rho_{0}$ of $H(x, \rho)$ must satisfy both equations (6.2).

We shall next restate Theorem 6.2 in terms of the value distributions of $H(x, \rho)$, as follows:

THEOREM 6.3. Let $H(x, \rho)$ be an axisymmetric harmonic polynomial, $h(\zeta)$ its associate and $\gamma$ any real constant. In $C$ let $\kappa$ be a convex region which is symmetric in the axis of reals and contains all the zeros of $h(\zeta)-\gamma$. Let $\kappa_{0}$ be the convex hull of the set $\{0, \kappa\}$. Then in the notation of Theorem 2.2 no critical circle $x=x_{0}, \rho=\rho_{0}$ of $H(x, \rho)$ can lie in any of the cones $K_{j}(\kappa, n-1), K_{j}\left(\kappa_{0}, n\right), j=1$ or 2.

Proof. Let us apply Lucas' Theorem [3, p. 22] which states that the zeros of the derivative of a polynomial $f$ in a complex variable lie in the convex hull of the zeros of $f$. This implies that $\kappa^{\prime} \subset \kappa, \kappa_{0}^{\prime} \subset \kappa_{0}$, and that therefore Theorem 6.3 follows from Theorem 6.2.

REMARK. Theorem 2.2 throws some light upon the location of the level surface $H(x, \rho)=\gamma$ of the harmonic polynomial $H(x, \rho)$ whereas Theorem 6.3 indicates the circles $x=x_{0}, \rho=\rho_{0}$ along which the level surface may consist of more than one analytic regular surface element [6, pp. 273-276]. 
7. Critical circles of harmonic functions with rational associates. As a generalization of Theorem 6.3 we propose the following result:

THEOREM 7.1. Let $\Psi(x, \rho)$ be an axisymmetric harmonic function whose associate is a rational function $\psi(\zeta)=p(\zeta) / q(\zeta)$. Let us denote by $n$ and $m$ the degrees of the polynomials $q(\zeta)$ and $\left[q(\zeta)^{2} \psi^{\prime}(\zeta)\right]$ respectively. Let $\kappa$, a convex region symmetric in the real axis of the $\zeta$-plane, contain all finite poles of $\psi(\zeta)$ and also all its finite critical points. Let $\kappa_{0}$ be the convex hull of the set $(0, \kappa)$. Then in the notation of Theorem 2.2, no critical circle of $\Psi(x, \rho)$ lies in any of the cones $K_{j}(\kappa, 2 n+m), K_{j}\left(\kappa_{0}, 2 n+m+1\right)$, $j=1$ or 2 .

Proof. If $\nabla \Psi=0$ for $x=x_{0}, \rho=\rho_{0}$, then also $\partial \Psi / \partial x=0$ and $r \partial \Psi / \partial r=0$ for $x=x_{0}$, $\rho=\rho_{0}$. Now, $\partial \Psi / \partial x$ and $r \partial \Psi / \partial r$ are given by expressions obtained by replacing $H$ by $\Psi$ and $h$ by $\psi$ in equations (5.4) and (5.6). Such expressions reveal $\partial \Psi / \partial x$ and $r \partial \Psi / \partial r$ to be axisymmetric harmonic functions whose associates are $\psi^{\prime}(\zeta)$ and $\zeta \psi^{\prime}(\zeta)$ respectively. If now we apply Theorem 4.2 to each of these functions and require $\partial \Psi / \partial x=0$ and $\partial \Psi / \partial r=0$ simultaneously, we thereby obtain Theorem 7.1.

We next consider the location of the critical circles of $\Psi(x, \rho)$ in relation not to poles and critical points of its associate $\psi(\zeta)$ but to the poles and zeros of $\psi(\zeta)$. To aid our work, we shall make use of Bôcher's Theorem [3, p. 94] which states that, if $p(\zeta)$ and $q(\zeta)$ are both polynomials of degree $n$ and if the zeros of $p$ are in a circular domain $D_{1}$ and those of $q$ in a circular domain $D_{2}$ where $D_{2} \cap D_{1}=\varnothing$, then all the critical points of $\psi(\zeta)=p(\zeta) / q(\zeta)$ lie in $D_{1} \cup D_{2}$.

THEOREM 7.2. In $C$ let $\omega_{1}$ and $\omega_{2}$ be two circular disks with centers on the real axis and such that $\mathscr{R}\left(\zeta_{1}\right)<\mathscr{R}\left(\zeta_{2}\right)$ for all $\zeta_{1} \in \omega_{1}$ and $\zeta_{2} \in \omega_{2}$. Let $\Psi(x, \rho)$ be a harmonic function with a rational associate $\psi(\zeta)$ possessing exactly $n$ finite zeros and $n$ finite poles both counted with multiplicities. Suppose that the zeros of $\psi(\zeta)$ lie in $\omega_{1}$ and the poles of $\psi(\zeta)$ in $\omega_{2}$. Suppose that $\psi$ has $m$ finite critical points $(m \leqq 2 n-1), k$ of which lie in $\omega_{1}$ and that

$$
\Omega=K_{2}\left(\omega_{1}, \mu\right) \cap K_{1}\left(\omega_{2}, \nu\right) \neq \varnothing
$$

for some choice of positive numbers $\mu, \nu$ with

$$
\lambda=(k / \mu)+[(m-k+2 n) / \nu]<1 .
$$

Then no circle $x=x_{0}, \rho=\rho_{0}$ in $\Omega$ can be a critical circle of $\Psi(x, \rho)$.

Proof. Let us set

$$
q(\zeta)^{2} \psi^{\prime}(\zeta)=\prod_{j=1}^{m}\left(\zeta-\alpha_{j}\right), \quad q(\zeta)=\prod_{j=1}^{n}\left(\zeta-\beta_{j}\right) .
$$

If $\alpha_{j} \in \omega_{1}$ for $j=1,2, \ldots, k$, then by Bôcher's Theorem, $\alpha_{j} \in \omega_{2}$ for $j=k+1, k+2$, $\ldots, m$. Hence, the $\alpha_{j}, j=1,2, \ldots, k$ satisfy the inequality (4.8), whereas the $\beta_{j}$ (and the remaining $\alpha_{j}$ ) satisfy inequality (4.11). Setting

$$
w(t)=\prod_{j=1}^{m}\left(\alpha_{j}-x_{0}-i \rho_{0} \cos t\right) / \prod_{j=1}^{n}\left(\beta_{j}-x_{0}-i \rho_{0} \cos t\right)^{2},
$$


we find that

$$
k \pi-\lambda \pi / 2<\arg w<k \pi+\lambda \pi / 2 .
$$

Hence, $\int_{0}^{2 \pi} w(t) d t \neq 0$, which implies that for the given $x=x_{0}, \rho=\rho_{0}, \partial \Psi / \partial x \neq 0$ and thus that Theorem 7.2 is valid.

To choose a circle $x_{0}=x_{0}, \rho=\rho_{0}$ for which $\partial \Psi / \partial r \neq 0$, we denote by $\kappa_{0}$ the convex hull of the set $\left(0, \omega_{1}\right)$ and select positive constants $\mu_{0}, \nu_{0}$ such that

$$
\lambda_{0}=\left[(k+1) / \mu_{0}\right]+\left[(m-k+2 n) / v_{0}\right]<1
$$

and such that

$$
\Omega_{0}=K_{2}\left(\kappa_{0}, \mu_{0}\right) \cap K_{1}\left(\omega_{2}, \nu_{0}\right) \neq \varnothing .
$$

Now, by reasoning similar to that leading to Theorem 7.2 we conclude that $\partial \Psi / \partial r \neq 0$ along such a circle. We thus are led to the result

THEOREM 7.3. Theorem 7.2 remains valid if $\lambda$ in (7.2) is replaced by $\lambda_{0}$ in (7.3) and at the same time $\Omega$ in (7.1) is replaced by $\Omega_{0}$ in (7.4).

8. Extension to certain other harmonic functions. We shall next study harmonic polynomials of the form

$$
F(x, y, z)=\sum_{j=J}^{n} A_{j} r^{j} P_{j}^{m(n-j)}(x / r) \cos m(n-j) \phi
$$

where $m$ is some given integer, $J \geqq[m n /(m+1)]$ and $P_{j}^{k}(\cos \phi)$ is the "associated Legendre function" [5, p. 323]. Clearly, $F(x, y, z)$ is not axisymmetric. Let us begin by setting up an integral representation for $F(x, y, z)$ in the form (1.5).

THEOREM 8.1. The harmonic functions $F(x, y, z)$ in (8.1) may be represented in the form (1.5) in which the associate is

$$
f(\zeta, \tau)=\tau^{-m n} \psi\left(\tau^{m} \zeta\right)
$$

where

$$
\psi(\zeta)=\sum_{j=\mathrm{J}}^{n} a_{j} \zeta^{j}, \quad a_{j}=\{[j+m(n-j)] ! / j !\} A_{j}
$$

and $\zeta$ is given by (1.6) or, as $\tau=e^{t i}$, equivalently by

$$
\zeta=x+i(y \cos t+z \sin t)=x+i \rho \cos (t-\phi) .
$$

That is,

$$
F(x, y, z)=(1 / 2 \pi) \int_{0}^{2 \pi} e^{-m n t i} \psi\left(e^{m t i}[x+i \rho \cos (t-\phi)]\right) d t
$$


Proof. To establish Theorem 8.1, we shall use the Laplace integral for $P_{j}^{k}(x / r)$ which we may write as follows $[5$, p. 326]:

$$
\begin{aligned}
r^{j} P_{j}^{k}(x / r) & =[(j+k) ! /(j ! 2 \pi)] \int_{0}^{2 \pi}(x+i \rho \cos t)^{j} e^{-k t i} d t \\
& =[(j+k) ! /(j ! \pi)] \int_{0}^{\pi}(x+i \rho \cos t)^{j} \cos k t d t .
\end{aligned}
$$

Writing

$$
\int_{0}^{2 \pi}[x+i \rho \cos (t-\phi)]^{j} e^{-k t i} d t=e^{-k \phi i} \int_{0}^{2 \pi}(x+i \rho \cos t)^{j} e^{-k t i} d t,
$$

we find that

$$
[(j+k) ! / j !] \int_{0}^{2 \pi}[x+i \rho \cos (t-\phi)]^{j} e^{-k t i} d t=2 \pi r^{j} P_{j}^{k}(x / r) e^{-k \phi i}
$$

Using (8.3) and (8.8), we may now evaluate the integral in (8.6) as follows:

$$
\begin{aligned}
F(x, y, z) & =(1 / 2 \pi) \int_{0}^{2 \pi} e^{-m n t i} \sum_{j=J}^{n} a_{j} e^{j m t i}[x+i \rho \cos (t-\phi)]^{j} d t \\
& =\sum_{j=J}^{n}\{(j !) /[j+m(n-j)] !\} a_{j} r^{j} P_{j}^{m(n-j)}(x / r) \cos [m(n-j) \phi] .
\end{aligned}
$$

Thus, (8.6) is a valid representation for (8.1).

As for the value distributions of $F(x, y, z)$, we have the following theorem:

THEOREM 8.2. Let $F(x, y, z)$ be the harmonic function (8.1) and $f(\zeta, \tau)$ its associate (8.3). If all the zeros of $\psi$ lie in the disk $\omega:|\zeta| \leqq a$, then no point of the level surface $F(x, y, z)=0$ lies in the cones

$$
0<\rho \leqq \pm x \tan (\pi / 2 n)-a \sec (\pi / 2 n) .
$$

Proof. Let us factor $\psi(\zeta)$ as $\psi(\zeta)=a_{n} \zeta^{J} \Pi_{j=1}^{n-J}\left(\zeta-\alpha_{j}\right)$. Then

$$
\begin{aligned}
w(t) & =e^{-m n t i} \psi\left(e^{m t i}[x+i \rho \cos (t-\phi)]\right) \\
& =e^{-m n t i} e^{J m t i}[x+i \rho \cos (t-\phi)]^{J} \prod_{j=1}^{n-J}\left\{e^{m t i}[x+i \rho \cos (t-\phi)]-\alpha_{j}\right\} \\
& =[x+i \rho \cos (t-\phi)]^{J} \prod_{j=1}^{n-J}\left[x+i \rho \cos (t-\phi)-\alpha_{j} e^{-m t i}\right] .
\end{aligned}
$$

Since by hypothesis $\left|\alpha_{j}\right| \leqq a$, also $\left|\alpha_{j} e^{-m t i}\right| \leqq a$ for $j=1,2, \ldots, n-J$ and hence by the same reasoning as we used for proving Theorems 2.1 and 2.2, we infer from (8.6) that $F(x, y, z) \neq 0$ in the two cones $K_{1}(\omega, n)$ and $K_{2}(\omega, n)$, thereby completing the proof of Theorem 8.2.

Let us next obtain some necessary conditions on the location of the critical points of the function $F(x, y, z)$ given in (8.1). We shall prove the following 
THEOREM 8.3. Let all the zeros of polynomial (8.4) lie in the disk $\omega:|\zeta| \leqq a$. Then no critical circle of function (8.1) can lie in any of the cones

$$
K_{k}(\omega, n-j): 0<\rho \leqq(-1)^{k} x \tan [\pi / 2(n-j)]-a \sec [\pi / 2(n-j)],
$$

where $j=0,1 ; k=1,2$.

Proof. Using (8.5) let us differentiate the expression (8.6). Thus

$$
\begin{aligned}
\partial F / \partial x= & (1 / 2 \pi) \int_{0}^{2 \pi} e^{-m(n-1) t i} \psi^{\prime}\left(e^{m t i} \zeta\right) d t, \\
r(\partial F / \partial r) & =x(\partial F / \partial x)+y(\partial F / \partial y)+z(\partial F / \partial z) \\
& =(1 / 2 \pi) \int_{0}^{2 \pi} e^{-m(n-1) t i} \psi^{\prime}\left(e^{m t i} \zeta\right) \zeta d t .
\end{aligned}
$$

A necessary condition for a critical point $\left(x_{0}, y_{0}, z_{0}\right)$ is that $\nabla F=0$ and thus that $\partial F / \partial x=\partial F / \partial r=0$ at $\left(x_{0}, y_{0}, z_{0}\right)$. Comparing (8.10) and (8.11) with (8.6), we note that $\partial F / \partial x$ and $r(\partial F / \partial r)$ are harmonic polynomials whose associates have the form (8.3) with $\psi(\zeta)$ replaced by $\psi^{\prime}(\zeta)$ and $\zeta \psi^{\prime}(\zeta)$ respectively. Hence, Theorem 8.3 follows from Theorem 8.2, twice applied.

9. Axisymmetric harmonic functions in $R^{N}$. We now study harmonic functions $F$ in the $N$ real variables $x_{1}, x_{2}, \ldots, x_{N}$ in the case that $F$ depends only upon the variables $x$ and $\rho$ where

$$
x=x_{1}, \quad \rho^{2}=x_{2}^{2}+x_{3}^{2}+\cdots+x_{N}^{2} .
$$

Laplace's equation $\nabla^{2} F=0$ then reduces to the form

$$
(\partial / \partial x)\left(\rho^{N-2} \partial F / \partial x\right)+(\partial / \partial \rho)\left(\rho^{N-2} \partial F / \partial \rho\right)=0 .
$$

If we introduce polar coordinates

$$
x=r \cos \theta, \quad \rho=r \sin \theta,
$$

we find (9.2) to have the polynomial solution

$$
H(x, \rho)=\sum_{j=0}^{n} A_{j} r^{j} P_{j}^{(\mu)}(x / r), \quad 2 \mu=N-2,
$$

where $P_{n}^{(\mu)}(\cos \theta)$ is the Gegenbauer polynomial, the special case of the Jacobi polynomial $P^{(\alpha, \beta)}(\cos \theta)$ with $\alpha=\beta=(N-3) / 2=\mu-\frac{1}{2}$.

THEOREM 9.1. Every axisymmetric harmonic polynomial $H(x, \rho)$ of form $(9.4)$ may be written as

$$
H(x, \rho)=\left[2^{3-N} / \Gamma(\mu)^{2}\right] \int_{0}^{\pi} h(x+i \rho \cos t) \sin ^{N-3} t d t
$$

in terms of its associate

$$
h(\zeta)=\sum_{j=0}^{n} a_{j} \zeta^{j}, \quad a_{j}=[\Gamma(j+2 \mu) / j !] A_{j} .
$$


Proof. We may deduce (9.5) almost immediately from the expression [2, p. 167]

$$
r^{n} P_{n}^{(\mu)}(\cos \theta)=\frac{2^{1-2 \mu} \Gamma(n+2 \mu)}{n ! \Gamma(\mu)^{2}} \int_{0}^{\pi}(x+i \rho \cos t)^{n} \sin ^{2 \mu-1} t d t .
$$

Let us examine formula (9.5) with a view of determining the value distribution of the polynomial $H(x, \rho)$ in $N$-dimensional space.

THEOREM 9.2. Let $H(x, \rho)$ be the $N$-dimensional axisymmetric harmonic function (9.4) and $h(\zeta)$ its associate (9.6). Let $\gamma$ be an arbitrary real constant and let

$$
\gamma_{0}=\left[2^{N-3} \Gamma(\mu)^{2} / \lambda_{N}\right] \gamma
$$

where

$$
\lambda_{N}=\int_{0}^{\pi / 2} \sin ^{2 \mu-1} t d t=(\sqrt{ } \pi / 2)\left[\Gamma(\mu) / \Gamma\left(\mu-\frac{1}{2}\right)\right] .
$$

If $h(\zeta) \neq \gamma_{0}$ in the complement of a bounded axiconvex region $\omega$, then $H(x, \rho) \neq \gamma$ in either $N$-dimensional cone

$$
K_{j}(\omega, n): 0<\rho \leqq(-1)^{j}\left(x_{1}-c_{j}\right) \tan (\pi / 2 n),
$$

with $j=1,2$ and $\rho^{2}=x_{2}^{2}+x_{3}^{2}+\cdots+x_{N}^{2}$.

Proof. Let us note that

$$
H(x, \rho)-\gamma=\left[2^{N-3} \Gamma(\mu)^{2}\right]^{-1} \int_{0}^{\pi}\left[h(x+i \rho \cos t)-\gamma_{0}\right] \sin ^{2 \mu-1} t d t .
$$

Let us write

$$
h(\zeta)-\gamma_{0}=a_{n} \prod_{j=1}^{n}\left(\zeta-\alpha_{j}\right), \quad \alpha_{j} \in \omega
$$

Since $\sin ^{2 \mu-1} t>0$ for $0<t<\pi$, the reasoning proceeds as in previous sections and leads to a proof of Theorem 9.2.

Finally, regarding the critical points of $H(x, \rho)$ in $N$-dimensions, we can state the following

THEOREM 9.3. Let $H(x, \rho)$ be the $N$-dimensional axisymmetric polynomial given by (9.4) and $h(\zeta)$ be the polynomial given by (9.6). In $C$ let the convex region $\kappa$ be symmetric in the real axis and contain all the points where $h(\zeta)=\gamma_{0}$ for some real constant $\gamma_{0}$ and let $\kappa_{0}$ be the convex hull of set $\{0, \kappa\}$. Then no locus $x=x_{0}, \rho=\rho_{0}$ in $N$ dimensions can be a critical locus of $H(x, \rho)$ if it lies in any of the $R^{N}$ cones $K_{j}(\kappa, n-1), K_{j}\left(\kappa_{0}, n\right), j=1$ or 2 .

The proof of Theorem 9.3 is left to the reader, as is the verification that the formulas and theorems of this section reduce to those of $\S \S 2$ and 6 when $N=3$.

10. Application to axisymmetric harmonic vectors. By an axisymmetric harmonic vector $\boldsymbol{H}(x, \rho)$ we mean one that is continuous together with its first and second 
partial derivatives, in an axisymmetric region $\Omega$ and is such that

$$
\nabla \cdot \boldsymbol{H}(x, \rho)=0, \quad \nabla \times \boldsymbol{H}(x, \rho)=0, \quad(x, \rho) \subset \Omega .
$$

If we write $i_{x}$ and $i_{\rho}$ for the unit vectors in the positive $x$ and $\rho$ directions and

$$
\boldsymbol{H}(x, \rho)=P(x, \rho) i_{x}-\rho^{-1} S(x, \rho) i_{\rho},
$$

then $P(x, \rho)$ and $S(x, \rho)$ are respectively the potential and Stoke's Stream Function of some axisymmetric flow. The critical circles of $P$ are the stagnation circles in the flow. In an earlier paper [7] we showed that, if $p(\zeta)$ is the associate of $P(x, \rho)$, then

$$
S(x, \rho)=-(\rho i / 2 \pi) \int_{0}^{2 \pi} p(x+i \rho \cos t) \cos t d t .
$$

We note that $\left\{\rho^{-1} S(x, \rho) e^{i \theta}\right\}$, but not $S(x, \rho)$, is harmonic. When $P(x, \rho)$ is an axisymmetric harmonic polynomial, we shall refer to $\boldsymbol{H}(x, \rho)$ as an axisymmetric harmonic polynomial vector. Concerning such a vector we now prove the following

THEOREM 10.1. Let $\boldsymbol{H}(x, \rho)$ in (10.2) be an axisymmetric harmonic polynomial vector whose component $P(x, \rho)$ has an nth degree polynomial $p(\zeta)$ as its associate. Let $\gamma$ be a real constant and $\kappa$ a convex region in $C$ symmetric in the real axis and containing all the points $\zeta$ where $p(\zeta)=\gamma$. Let $\kappa_{0}$ be the convex hull of the set $\{0, \kappa\}$. Then no circle $\left(x_{0}, \rho_{0}\right)$ along which $\boldsymbol{H}(x, \rho)=\gamma i_{x}$ may lie in either of the regions

$$
\Omega_{j}=K_{j}(\kappa, n) \cap K_{j}\left(\kappa_{0}, n+1\right), \quad j=1,2,
$$

where $K_{j}(\omega, n)$ are the cones defined in (2.8) and (2.9).

Proof. The desired circles $\left(x_{0}, \rho_{0}\right)$ are the intersection of the two orthogonal surfaces of revolution

$$
P(x, \rho)=\gamma, \quad S(x, \rho)=0 .
$$

On setting $q(\zeta)=p(\zeta)-\gamma$, we may write

$$
\begin{aligned}
P(x, \rho)-\gamma & =(1 / 2 \pi) \int_{0}^{2 \pi} q(x+i \rho \cos t) d t \\
S(x, \rho) & =-(\rho i / 2 \pi) \int_{0}^{2 \pi} q(x+i \rho \cos t) \cos t d t \\
& =[P(x, \rho)-\gamma] x-(1 / 2 \pi) \int_{0}^{2 \pi} q(x+i \rho \cos t)(x+i \rho \cos t) d t .
\end{aligned}
$$

Hence, $\left(x_{0}, \rho_{0}\right)$ satisfy $(10.4)$ if simultaneously

$$
\begin{aligned}
\int_{0}^{2 \pi} q\left(x_{0}+i \rho_{0} \cos t\right) d t & =0, \\
\int_{0}^{2 \pi} q\left(x_{0}+i \rho_{0} \cos t\right)\left(x_{0}+i \rho_{0} \cos t\right) d t & =0 .
\end{aligned}
$$

However, equations (10.5) take on the same form as (6.2) if we replace $h^{\prime}(\zeta)$ and $\zeta h^{\prime}(\zeta)$ occurring in (5.4) and (5.6) by $q(\zeta)$ and $\zeta q(\zeta)$ respectively. If we also let $\kappa$ and $\kappa_{0}$ replace the $\kappa^{\prime}$ and $\kappa_{0}^{\prime}$ given in Theorem 6.2, we thereby derive Theorem 10.1. 


\section{REFERENCES}

1. Stefan Bergman, Integral operators in the theory of linear partial differential equations, Ergebnisse der Mathematik und ihrer Grenzgebiete, N.F., Heft 23, Springer-Verlag, Berlin, 1961. MR 25 \#5277.

2. Robert P. Gilbert, Function theoretic methods in partial differential equations, Math. in Sci. and Engineering, vol. 54, Academic Press, New York, 1969. MR 39 \#3127.

3. Morris Marden, Geometry of polynomials, Math. Surveys, no. 3, Amer. Math. Soc., Providence, R. I., 1966. MR 37 \#1562.

4. Serge Bernstein, Leçons sur propriétés extrémales et la meilleure approximation des fonctions analytiques d'une variable réelle, Gauthier-Villars, Paris, 1926.

5. E. T. Whittaker and G. N. Watson, $A$ course of modern analysis, Cambridge Univ. Press, New York, 1943.

6. Oliver Kellogg, Foundations of potential theory, Springer-Verlag, Berlin, 1929.

7. Morris Marden, Axisymmetric harmonic vectors, Amer. J. Math. 67 (1945), 109-122. MR 7, 225.

UNIVERSITY OF WISCONSIN-MILWAUKEE, MilWAUKeE, WisCoNSIN 53201 